\title{
Fatty acids and their role in type-2 diabetes (Review)
}

\author{
SHILPA S. SHETTY ${ }^{1}$ and SUCHETHA KUMARI ${ }^{2}$ \\ ${ }^{1}$ Central Research Laboratory, ${ }^{2}$ Department of Biochemistry, K.S. Hegde Medical Academy, \\ Nitte (Deemed to be University), Deralakatte, Mangalore, Karnataka 575018, India
}

Received September 4, 2020; Accepted February 15, 2021

DOI: $10.3892 /$ etm.2021.10138

\begin{abstract}
Age, lifestyle and diet are major risk factors for the onset of type 2 diabetes mellitus (T2DM). Insulin resistance (IR) and $\beta$-cell dysfunction underlie the pathophysiology of T2DM. Diabetic populations are also prone to lipid and lipoprotein abnormalities as an indirect effect of IR on key metabolic enzymes. However, recent studies suggested that lipid changes may not only be a consequence of impaired glucose metabolism but also a causative factor. Fatty acids (FAs) influence translocation of glucose transporters and insulin receptor binding and signalling, in addition to cell membrane fluidity and permeability. It is thus suggested that FAs may have an essential role in the development of IR and T2DM. Specific combinations of FAs within phospholipids and triglycerides were indicated to exhibit the strongest associations with the risk of T2DM. The aim of the present review was to investigate the role of FAs in the pathogenesis of T2DM, as it has yet to be fully elucidated.
\end{abstract}

\section{Contents}

1. Introduction

2. FAs

3. n-3 and n-6 PUFA bioactive mediators

4. FAs and T2DM

5. Conclusion

\section{Introduction}

Type 2 diabetes mellitus (T2DM) is characterized by insulin resistance (IR). Improved risk prediction and understanding of the pathogenesis underlying IR are crucial for the management of T2DM. The aetiology of IR is multifactorial. Genetic background and environmental factors, such as age, lifestyle and

Correspondence to: Dr Shilpa. S. Shetty, Central Research Laboratory, K.S. Hegde Medical Academy, Nitte (Deemed to be University), Deralakatte, Mangalore, Karnataka 575018, India E-mail: shilpajshetty@nitte.edu.in

Key words: diabetes, fatty acids, lipidomics diet, are two classifiable disease risk factors and the interaction between them contributes to the development of T2DM. Fatty acids (FAs) may have a key role in the development of IR and T2DM (1-3). However, the long-term effect of FAs on T2DM has yet to be fully elucidated (4).

While genomics, transcriptomics and proteomics have been widely used to improve the current understanding of obesity and T2DM, lipidomics is a tool that has been used comparatively less frequently (5). Lipidomics, which is a subcategory of metabolomics, may enhance the understanding of the contribution of FAs and lipids towards the development of health-related complications, particularly IR and T2DM. Circulating lipids and FAs may reflect an individual's lifestyle (e.g., diet and exercise) and their gene and protein activity, all of which may affect the development of IR and T2DM $(6,7)$. Lipidomic techniques have provided valuable information on obesity and T2DM-related changes in adipocyte (8), macrophage (9), skeletal muscle (10), lipoprotein lipid composition (11) and liver FA metabolism (12), and have allowed researchers to better understand the contribution of obesity towards the development of T2DM. These methods have also enabled researchers to unravel the underlying mechanisms through which exercise, metformin and rosiglitazone improve the status of patients with T2DM $(12,13)$. Lipidomic techniques have revealed that quantifying specific FAs within lipid fractions [e.g., triglycerides (TGs) and phospholipids (PLs)] may provide a more accurate indication of IR and T2DM (13-15). It was demonstrated that specific combinations of FAs within PL and TG exhibited the strongest association with the risk of T2DM, particularly shorter saturated FAs (SFAs) (15).

\section{FAs}

FAs may exist as free FAs (FFAs) in the body or they may combine with other molecules to form lipids, such as cholesterol esters (CEs), PLs and TGs (16-20). Low-density lipoprotein (LDL), very LDL and high-density lipoprotein consist of different relative amounts of CE, PL and TG. PLs have a hydrophilic phosphate head and two hydrophobic FA tails linked to a glycerol molecule. TGs contain FFAs that are esterified to a glycerol molecule. TG is the main form of fat in the diet. CEs are formed from FA and cholesterol by an ester bond between the carboxylate group of FAs and the hydroxyl group of cholesterol (16-20).

In addition to using fat as an energy source, the human body also synthesizes, desaturates and elongates FAs 
endogenously (21). The synthesis of FAs is low if the dietary intake of fat is moderate or high, whereas a high dietary intake of carbohydrates stimulates de novo lipogenesis (22-24).

Several factors affect endogenous FA metabolism and plasma FA composition, such as age and sex, health status, epigenetic changes and genes. Desaturation and elongation are steps of a metabolic pathway in which dietary and endogenous SFAs are elongated and converted to monounsaturated FAs (MUFAs), while highly polyunsaturated FAs (PUFAs) are synthesized from dietary n-3 FAs (e.g., $\alpha$-linolenic acid) and n-6 FAs (e.g., linoleic acid) in the liver and adipose tissue $(25,26)$. Desaturases and elongases are enzymes that activate these metabolic pathways. Desaturases add a double bond to the FA, whereas elongases lengthen the FA by adding two carbon molecules to the carbon chain. The metabolism of PUFAs is important, as they are the essential FAs of the human body $(25,26)$.

A detailed understanding of the molecular structures and mechanisms implicated in FA synthesis and degradation may enable nutritional investigators to elucidate how and why specific dietary patterns and classes of FAs are associated with IR and T2DM (27). The changes in FA levels in the circulation caused by IR are summarized in Fig. 1. In a normal condition, when insulin binds to the insulin receptor it inactivates the enzyme hormone sensitive lipase (HSL) involved in the hydrolysis of TG to glycerol and FFA. In an insulin resistant state, there is an increase in the activity of HSL releasing free fatty acids into circulation to the liver. In the liver, hepatocytes take up the fatty acids and channel them into secretory pathways. The enzyme lipoprotein lipase in the blood vessels hydrolyses monoglycerides and FFA. As this cycling process continues, FFA also increases (Fig. 1).

Fig. 2 depicts how FFAs affect the insulin signalling pathway by increasing diacylglycerol (DAG), reactive oxygen species and protein kinase C (PKC), which in turn increases insulin receptor substrate-1 (IRS-1) serine phosphorylation and decreases IRS-1 tyrosine phosphorylation, thereby inhibiting the activity of PI3K. This finally leads to disturbance of the fragile balance between $\beta$-cell function and peripheral insulin resistance, which eventually results in the clinical manifestation of T2DM.

\section{3. n-3 and n-6 PUFA bioactive mediators}

Adipocytes and pancreatic $\beta$-cells produce a major group of pro-inflammatory mediators, the eicosanoids. Oxidation of n-3 and n-6 PUFAs [eicosapentaenoic acid (EPA), arachidonic acid (AA) and the linolenic acid (LA) derivative dihomo- $\gamma$-LA] release major eicosanoids, including prostaglandins (PGs), leukotrienes (LTs), thromboxanes and lipoxins. These may be either pro-inflammatory or anti-inflammatory $(28,29)$. PGs and LTs derived from AA are pro-inflammatory, whereas PGs and LTs derived from EPA are anti-inflammatory (Fig. 3) $(16,30,31)$.

\section{FAs and T2DM}

A number of physiological pathways are affected by SFAs $(32,33)$. Hepatic de novo lipogenesis occurs in response to a high dietary intake of carbohydrates or total calories, thereby increasing the endogenous levels of SFAs $(32,33)$. According to the Chinese philosophy concept, palmitic and oleic acid were described as the 'Yin' and 'Yang' of FAs by Palomer et al (34). Increased palmitic acid levels in subjects with diabetes may be explained via the enhancement of deleterious complex lipid synthesis, impairment of cellular organelle function and promotion of receptor-mediated inflammation $(35,36)$. The rate of FA delivery to non-adipose tissues (liver, muscle, heart and pancreatic islets) depends on the increased plasma non-esterified FA (NEFA) content, promoting lipotoxicity and lipoapoptosis. These conditions raise intracellular palmitic acid levels above their mitochondrial oxidation limit and are converted to harmful complex FA-derived lipids, such as DAG and ceramide.

A high intracellular DAG content activates PKC isoforms, followed by phosphorylation of insulin serine residue 1 , attenuating the insulin signalling pathway. Pro-inflammatory signalling cascade inhibitor and ceramide synthesis are, in turn, activated by PKC isoforms. Furthermore, ceramide also inhibits mitochondrial $\beta$-oxidation of FAs, induces endoplasmic reticulum (ER) stress and activates the NACHT, LRR and PYD domains-containing protein 3 inflammasome, a major instigator of inflammation (37). The perturbation of ER homeostasis by increased NEFA levels affects the lipid composition of ER due to lipid dysregulation, resulting in ER stress, which affects calcium signalling and attenuation of protein translation. Eventually, these modifications cause metabolic dysregulation and T2DM.

As mentioned above, according to the Chinese philosophy concept, if palmitate is the 'Yin' of FAs in T2DM, oleic acid is the 'Yang', as it elicits beneficial effects on insulin sensitivity (38). These beneficial effects of oleic acid are mediated by several mechanisms. Oleic acid reduces leukotriene B4 (LTB4) levels and increases insulin sensitivity (39). Oleic acid elicits anti-inflammatory effects by increasing the levels of the anti-inflammatory cytokine IL-10 and adiponectin and reducing the levels of pro-inflammatory cytokines (interleukin-6, tumour necrosis factor- $\alpha$ ), induces macrophage polarization and reduces the secretion of LTB4 (38). FFA receptor 4 or G protein-coupled receptor (GPR)120 may also mediate these effects of oleic acid.

Unsaturated FAs have a key role in normal tissue function as major components of membrane bilayers, determining factors of cell structure and function and modulators of gene expression. Unsaturated FAs may be MUFAs or PUFAs (38). A previous study demonstrated that, in subjects with high fasting TG levels, MUFAs buffer $\beta$-cell hyperactivity and IR (40).

LA and $\alpha$-LA (ALA) are essential FAs, as the human body lacks the desaturase enzymes catalysing their endogenous production and they may only be obtained from dietary sources $(32,36)$. The relative FA levels in the plasma, serum or erythrocyte membrane reflect the rate of conversion of different FAs catalysed by key enzymes, such as FA desaturases (FADS) and elongases $(33,34)$.

ALA is converted into the long-chain omega-3 polyunsaturated FA EPA or docosahexaenoic acid (DHA) (41). Preclinical data suggested that omega-3 improves insulin signalling (41). The proposed mechanism of how omega-3 improves insulin sensitivity is that omega-3 PUFAs attenuate ER stress, enhance FA $\beta$-oxidation in mitochondria and uncouple mitochondria, 
Normal state

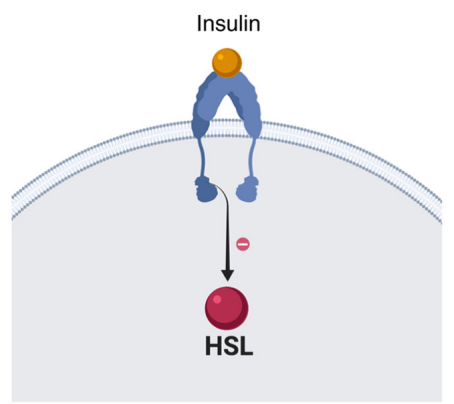

Insulin resistant state

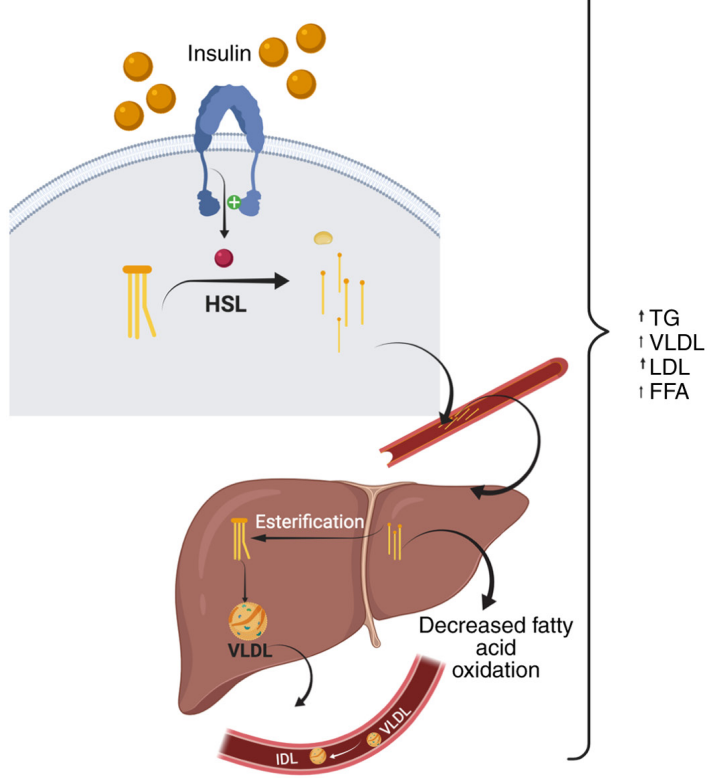

Figure 1. IR and changes in FA composition. In adipose tissue, in the normal state, insulin binds to the insulin receptor, inhibiting the enzyme HSL in the adipocytes of the adipose tissue. This HSL hydrolyses lipids such as TGs. In the state of IR, insulin is unable to bind to insulin receptors, thereby suppressing intracellular signals. In turn, it activates the HSL enzyme, which hydrolyse TGs to glycerol and FFAs, which are released into the circulation towards the liver. Liver hepatocytes take up FFAs to channel them to their secretory pathways. In the case of hyperinsulinemia/IR, esterification is increased. In the blood vessels, the enzyme LPL hydrolyses monoglycerides and FFAs. A certain proportion of these are delivered to liver LPL (hydrolysis). This process continues and more LDL and FFAs are formed (schematic created with BioRender.com). FFA, free fatty acid; HSL, hormone-sensitive lipase; LPL, lipoprotein lipase; LDL, low-density lipoprotein; VLDL, very low-density lipoprotein; TG, triglyceride; IR, insulin resistance.

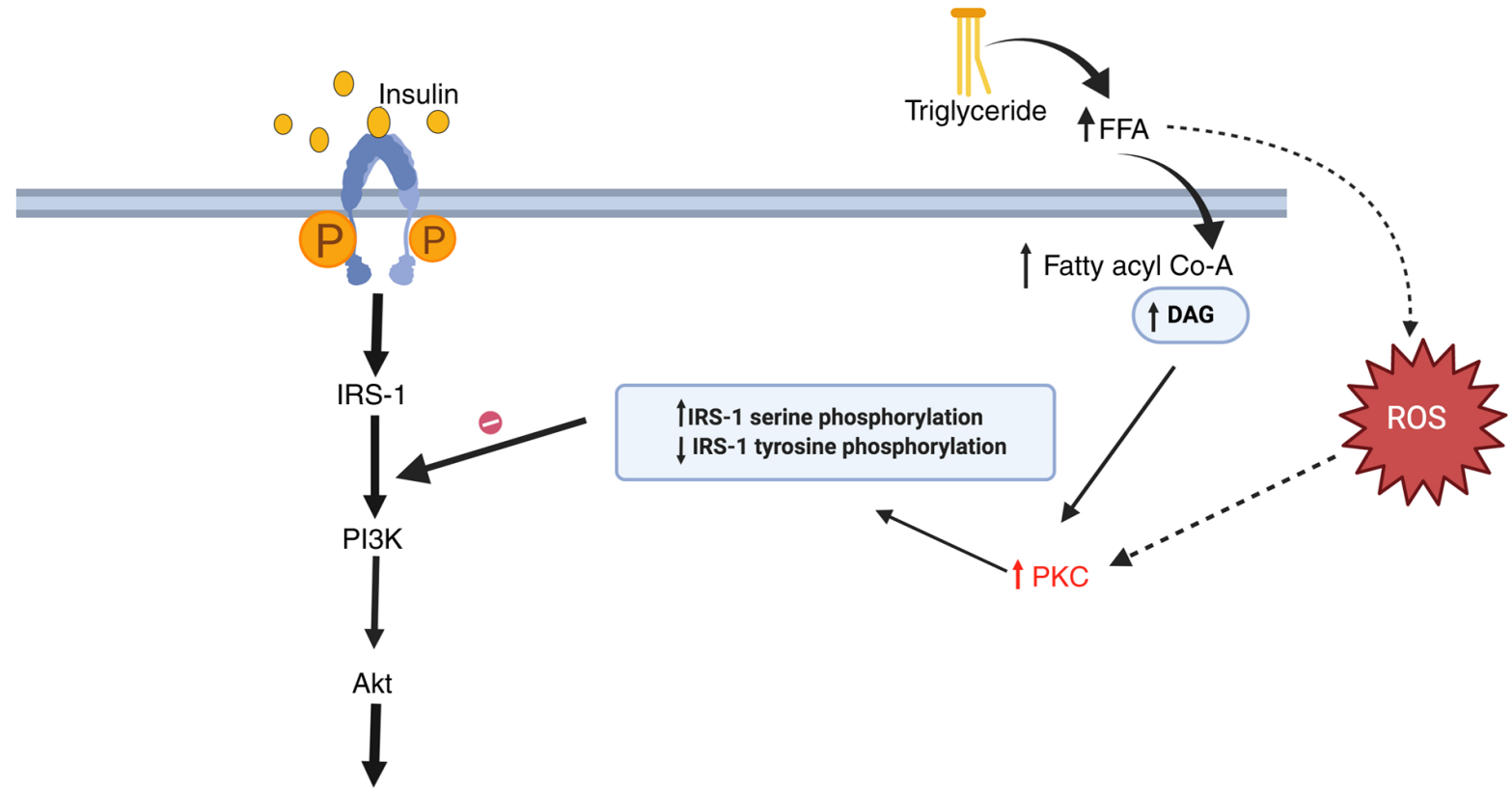

AS160

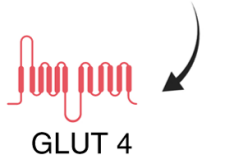

Figure 2. Effect of TGs and FFAs on the insulin signalling pathway. Elevated TG levels increase FFAs, which leads to accumulation of DAG and fatty acyl Co-A along with increased ROS. All of this together activates PKC, which interrupts the insulin signalling pathway (schematic created with BioRender. com). IRS, insulin receptor substrate; AS160, Akt substrate of $160 \mathrm{kDa}$; Glut-4, glucose transporter 4; FFA, free fatty acid; ROS, reactive oxygen species; P, phosphate; PKC, protein kinase C; DAG, diacylglycerol; Co-A, coenzyme A; FFA, free fatty acid. 

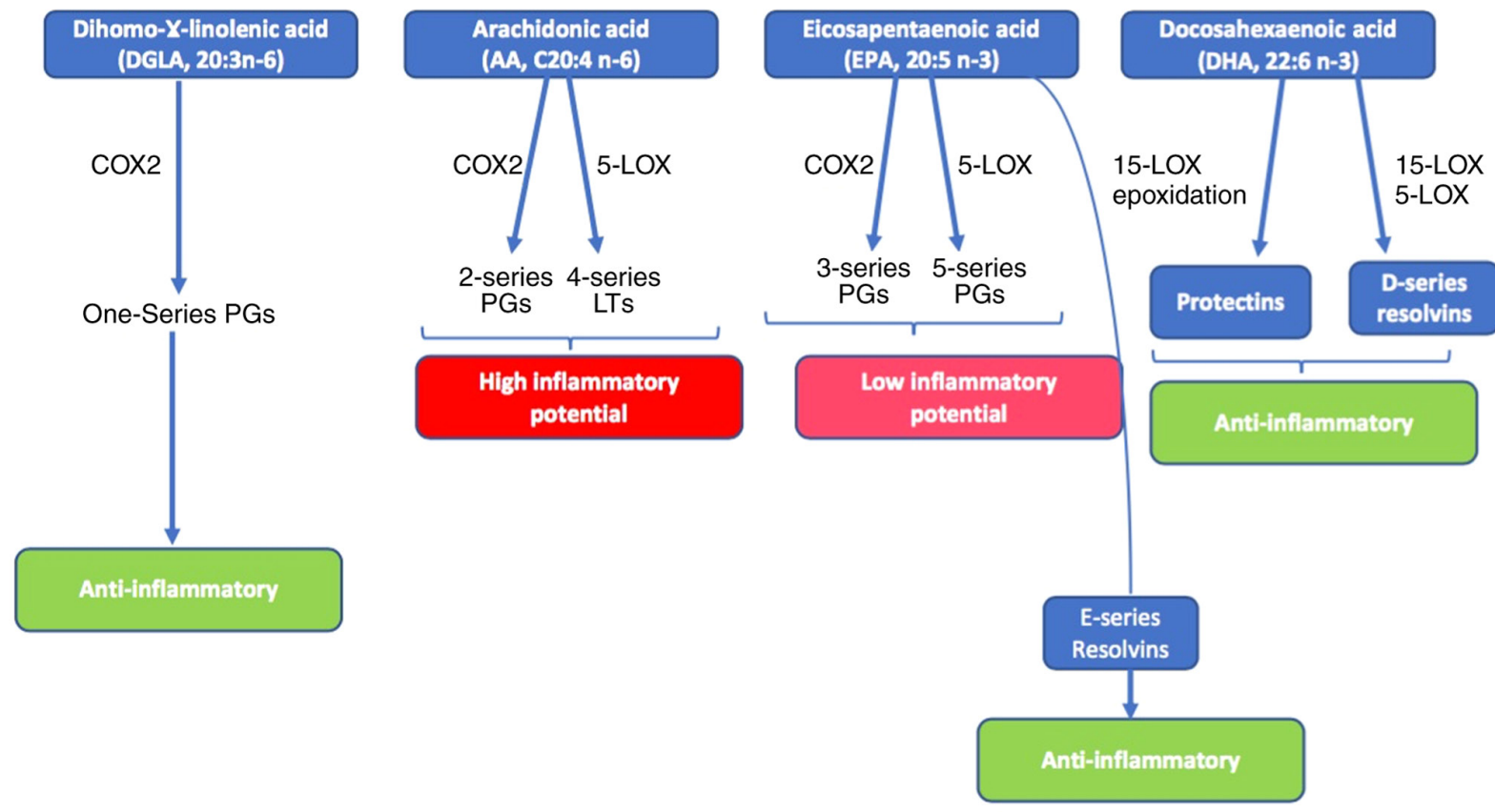

Figure 3. Bioactive mediators from omega-3 and omega-6 fatty acids. COX2, cyclooxygenase-2; LOX, lipoxygenase; PG, prostaglandin; LT, leukotriene.

causing reduction in lipid buildup and reactive oxygen species accumulation (38). In addition, a previous study reported the positive impact of these omega-3 FAs on mitofusin 2, which is involved in mitochondrial dynamics homeostasis and mitochondria-associated membrane integrity maintenance (41).

EPA and DHA regulate insulin sensitivity via Akt phosphorylation, activation of AMP-activated protein kinase (a treatment target for T2DM) and activation of peroxisome proliferator-activated receptor (PPAR)- $\gamma$ (42). Several studies have demonstrated the role of EPA and DHA in preventing lipotoxicity and insulin sensitivity restoration (43-48). Omega-3 FAs also modulate pancreatic $\beta$-cell insulin secretion by exerting a direct effect on the lipid raft function and structure, and indirectly by inhibition of the expression of pro-inflammatory mediators in adipose tissue and the promotion of adipokine production (48). By contrast, omega-6 FAs are pro-inflammatory, as the product of omega- 6 desaturation, AA, produces inflammatory cytokines and eicosanoids.

Omega-3 PUFAs inhibit inflammatory cytokine and eicosanoid production from AA and induce adipokine production from adipose tissue, and directly affect $\beta$-cell function by binding to PPARs, GPR40 and GPR120, thereby promoting insulin secretion (48). It remains elusive how exactly these PUFAs affect glucose metabolism (49). It has also been reported that a defect in the activity of D6 and D5 desaturases, the key enzymes of the PUFA desaturation pathway, may be a key factor in the development of IR (48), with ensuing public health implications.

Overall, the role of the increase in the FFA content of the body in IR may be explained with the insulin binding to its receptor. Under normal conditions, binding of insulin to its receptor in the adipose tissue cell membrane triggers an intracellular signal that suppresses the activity of hormone-sensitive lipase (HSL), an intracellular enzyme found in adipocytes, which hydrolyses lipids such as TG.
When an individual is insulin-resistant, this intracellular signalling is suppressed, thereby increasing HSL activity, which hydrolyses TG to glycerol and FFAs, which are then released into the circulation and accumulate in the liver. These FAs are taken up by the hepatocytes and are channelled to their secretory pathways. Due to IR, esterification increases. The enzyme lipoprotein lipase in the blood vessels hydrolyses monoglycerides and FFAs. This process continues, thereby increasing the FA content (49).

Omega-3 and omega-6 FAs continuously compete for the desaturation enzymes. ALA is preferred over LA by both FADS1 and FADS2 (50). Therefore, maintaining an optimal balance between omega- 6 and omega- 3 FAs in the diet is crucial for human health, as the physiological state is anti-inflammatory (51). Unbalanced omega-6/omega-3 ratio in favour of omega-6 PUFAs is highly prothrombotic and pro-inflammatory, contributing to the prevalence of atherosclerosis, obesity and diabetes (51). As previously reported, the target ratio of omega-6/omega-3 should be $1: 1$ to $2: 1$ (51). Although the Indian diet is low in fat when compared to the western diet (52), the incidence of coronary artery disease and $\mathrm{T} 2 \mathrm{DM}$ are on the increase in this population. As per the previous report by Mani and Kurpad (52) although the Indian diet is rich in PUFAs, the ratio is in favour of omega-6 FAs, since the most abundant PUFA in the majority of plant products is LA. Therefore, the LA intake is high, irrespective of the type of vegetable oil used in a typical Indian diet (52). Therefore, dietary FA interventions specifically aimed at maintaining an optimal omega-6/omega-3 ratio may overcome the risk of developing T2DM.

\section{Conclusion}

Taken together, the present review indicated that the desaturation pathway appears to be highly important for maintaining 
lipid homeostasis in the human body, the key to which may be ensuring a healthy omega-6/omega-3 ratio. This suggests that, in T2DM research, lipidomics may represent a useful approach to improve the understanding of the effect of different lipids on the risk of developing T2DM.

\section{Acknowledgements}

Not applicable.

\section{Funding}

No funding was received.

\section{Availability of data and materials}

Not applicable.

\section{Authors' contributions}

SSS and SK wrote, revised and edited the article. Both authors have read and approved the final manuscript.

\section{Ethics approval and consent to participate}

Not applicable.

\section{Patient consent for publication}

Not applicable.

\section{Competing interests}

The authors declare that they have no competing interests.

\section{References}

1. Boden G and Shulman GI: Free fatty acids in obesity and type 2 diabetes: Defining their role in the development of insulin resistance and $\beta$-cell dysfunction. Eur J Clin Invest 32 (Suppl 3): 14-23, 2002.

2. Boden G: Effects of free fatty acids (FFA) on glucose metabolism: Significance for insulin resistance and type 2 diabetes. Exp Clin Endocrinol Diabetes 111: 121-124, 2003.

3. Boden G: Obesity, insulin resistance and free fatty acids. Curr Opin Endocrinol Diabetes Obes 18: 139-143, 2011.

4. Risérus U, Willett WC and Hu FB: Dietary fats and prevention of type 2 diabetes. Prog Lipid Res 48: 44-51, 2009.

5. Shevchenko A and Simons K: Lipidomics: Coming to grips with lipid diversity. Nat Rev Mol Cell Biol 11: 593-598, 2010.

6. Gutch M, Kumar S, Razi SM, Gupta KK and Gupta A: Assessment of insulin sensitivity/resistance. Indian J Endocrinol Metab 19: 160-164, 2015.

7. Garaulet M, Pérez-Llamas F, Pérez-Ayala M, Martínez P, de Medina FS, Tebar FJ and Zamora S: Site-specific differences in the fatty acid composition of abdominal adipose tissue in an obese population from a Mediterranean area: Relation with dietary fatty acids, plasma lipid profile, serum insulin, and central obesity. Am J Clin Nutr 74: 585-591, 2001.

8. Pietiläinen KH, Róg T, Seppänen-Laakso T, Virtue S, Gopalacharyulu P, Tang J, Rodriguez-Cuenca S, Maciejewski A, Naukkarinen J, Ruskeepää AL, et al: Association of lipidome remodeling in the adipocyte membrane with acquired obesity in humans. PLoS Biol 9: e1000623, 2011.

9. Borkman M, Storlien LH, Pan DA, Jenkins AB, Chisholm DJ and Campbell LV: The relation between insulin sensitivity and the fatty-acid composition of skeletal-muscle phospholipids. N Engl J Med 328: 238-244, 1993.
10. Ståhlman M, Pham HT, Adiels M, Mitchell TW, Blanksby SJ, Fagerberg B, Ekroos K and Borén J: Clinical dyslipidemia is associated with changes in the lipid composition and inflammatory properties of apolipoprotein- B containing lipoproteins from women with type 2 diabetes. Diabetologia 55: 1156-1166, 2012.

11. Wang Y, Botolin D, Xu J, Christian B, Mitchell E, Jayaprakasam B, Nair MG, Peters JM, Busik JV, Olson LK, et al: Regulation of hepatic fatty acid elongase and desaturase expression in diabetes and obesity. J Lipid Res 47: 2028-2041, 2006.

12. Huo T, Cai S, Lu X, Sha Y, Yu M and Li F: Metabonomic study of biochemical changes in the serum of type 2 diabetes mellitus patients after the treatment of metformin hydrochloride. J Pharm Biomed Anal 49: 976-982, 2009.

13. Markgraf DF, Al-Hasani H and Lehr S: Lipidomics-Reshaping the Analysis and Perception of Type 2 Diabetes. Int J Mol Sci 17: $1841,2016$.

14. Wong G, Barlow CK, Weir JM, Jowett JB, Magliano DJ, Zimmet P, Shaw J and Meikle PJ: Inclusion of plasma lipid species improves classification of individuals at risk of type 2 diabetes. PLoS One 8: e76577, 2013.

15. Rhee EP, Cheng S, Larson MG, Walford GA, Lewis GD, McCabe E, Yang E, Farrell L, Fox CS, O'Donnell CJ, et al: Lipid profiling identifies a triacylglycerol signature of insulin resistance and improves diabetes prediction in humans. J Clin Invest 121: 1402-1411, 2011

16. Vargas E and Sepulveda CMA: Biochemistry, Insulin Metabolic Effects. In: StatPearls. Stat Pearls Publishing, Treasure Island, FL, 2019. https://www.ncbi.nlm.nih.gov/books/NBK525983/. Accessed April 21, 2019.

17. Betts GJ, Desaix P, Johnson E, Korol O, Kruse D, Poe B, et al: Human Anatomy and Physiology. OpenStax College, 2013.

18. Röder PV, Wu B, Liu Y and Han W: Pancreatic regulation of glucose homeostasis. Exp Mol Med 48: e219, 2016.

19. Tokarz VL, MacDonald PE and Klip A: The cell biology of systemic insulin function. J Cell Biol 217: 2273-2289, 2018.

20. Samuel VT and Shulman GI: Mechanisms for insulin resistance: Common threads and missing links. Cell 148: 852-871, 2012.

21. No authors listed: Diabetes is more prevalent among the urban poor: A summary of the findings of the ICMR-INDIAB Study. Curr Med Issues 15: 243-244, 2017.

22. Mohan V and Pradeepa R: Epidemiology of Diabetes in different regions of India. Health Administrator XXII 1, 2: 1-18, 2009.

23. Tandon N, Anjana RM, Mohan V, Kaur T, Afshin A, Ong K, Mukhopadhyay S, Thomas N, Bhatia E, Krishnan A, et al: The increasing burden of diabetes and variations among the states of India: The Global Burden of Disease Study 1990-2016 India State-Level Disease Burden Initiative Diabetes Collaborators. Lancet Glob Health 6: 1352-1362, 2018.

24. Chawla A, Chawla R and Jaggi S: Microvasular and macrovascular complications in diabetes mellitus: Distinct or continuum? Indian J Endocrinol Metab 20: 546-551, 2016.

25. Yajnik CS, Fall CH, Coyaji KJ, Hirve SS, Rao S, Barker DJ, Joglekar C and Kellingray S: Neonatal anthropometry: The thin-fat Indian baby. The Pune Maternal Nutrition Study. Int J Obes Relat Metab Disord 27: 173-180, 2003.

26. Unnikrishnan RI, Rema M,Pradeepa R, Deepa M, Shanthirani CS, Deepa R and Mohan V: Prevalence and risk factors of diabetic nephropathy in an urban south Indian population. The Chennai Urban Rural Epidemiology Study (CURES-45). Diabetes Care 30: 2019-2024, 2007.

27. Tan SY and Merchant J: Frederick Banting (1891-1941): Discoverer of insulin. Singapore Med J 58: 2-3, 2017.

28. Pradeepa R, Rema M, Vignesh J, Deepa M, Deepa R and Mohan V: Prevalence and risk factors for diabetic neuropathy in an urban south Indian population: The Chennai Urban Rural Epidemiology Study (CURES-55). Diabet Med 25: 407-412, 2008.

29. Mohan V, Deepa R, Rani SS, Premalatha G; Chennai Urban Population Study (CUPS No.5): Prevalence of coronary artery disease and its relationship to lipids in a selected population in south India: The Chennai Urban Population Study (CUPS No. 5). J Am CollCardiol 38: 682-687, 2001.

30. Premalatha G, Shanthirani CS, Deepa R, Markovitz J and Mohan V: Prevalence and risk factors of peripheral vascular disease in a selected south Indian population the Chennai Urban Population Study (CUPS). Diabetes Care 23: 1295-1300, 2000.

31. White MG, Shaw JA and Taylor R: Type 2 Diabetes: The Pathologic Basis of Reversible $\beta$-Cell Dysfunction. Diabetes Care 39: 2080-2088, 2016. 
32. Andersson-Hall U, Carlsson NG, Sandberg AS and Holmäng A Circulating Linoleic Acid is Associated with Improved Glucose Tolerance in Women after Gestational Diabetes. Nutrients 10: $1629,2018$.

33. Ertunc ME and Hotamisligil GS: Lipid signaling and lipotoxicity in metaflammation: Indications for metabolic disease pathogenesis and treatment. J Lipid Res 57: 2099-2114, 2016.

34. Palomer X,Pizarro-Delgado J,Barroso E and Vázquez-Carrera $\mathrm{M}$ : Palmitic and Oleic Acid: The Yin and Yang of Fatty Acids in Type 2 Diabetes Mellitus. Trends Endocrinol Metab 29: 178-190, 2018.

35. Wu HT, Chen W, Cheng KC, Ku PM, Yeh CH and Cheng JT: Oleic acid activates peroxisome proliferator-activated receptor $\delta$ to compensate insulin resistance in steatotic cells. J Nutr Biochem 23: 1264-1270, 2012.

36. Simopoulos AP: The impact of the Bellagio Report on healthy agriculture, healthy nutrition, healthy people: Scientific and policy aspects and the International Network of Centers for Genetics, Nutrition and Fitness for Health. J Nutrigenet Nutrigenomics 7: 191-211, 2014.

37. Linus Pauling Institute: Essential Fatty acids. https://lpi. oregonstate.edu/mic/other-nutrients/essential-fatty-acids\#membr ane-structure-function. Accessed May, 2019.

38. Lopez S, Bermudez B, Ortega A, Varela LM, Pacheco YM, Villar J, Abia R and Muriana FJ: Effects of meals rich in either monounsaturated or saturated fat on lipid concentrations and on insulin secretion and action in subjects with high fasting triglyceride concentrations. Am J Clin Nutr 93: 494-499, 2011.

39. Bhaswant M, Poudyal H and Brown L: Mechanisms of enhanced insulin secretion and sensitivity with n-3 unsaturated fatty acids. J Nutr Biochem 26: 571-584, 2015.

40. Lepretti M, Martucciello S, Burgos Aceves MA, Putti R and Lionetti L: Omega-3 Fatty Acids and Insulin Resistance: Focus on the Regulation of Mitochondria and Endoplasmic Reticulum Stress. Nutrients 10: 350, 2018.

41. Day EA, Ford RJ and Steinberg GR: AMPK as a therapeutic target for treating metabolic diseases. Trends Endocrinol Metab 28: 545-560, 2017.

42. Pérez-Matute P, Pérez-Echarri N, Martínez JA, Marti A and Moreno-Aliaga MJ: Eicosapentaenoic acid actions on adiposity and insulin resistance in control and high-fat-fed rats: Role of apoptosis, adiponectin and tumour necrosis factor-alpha. Br J Nutr 97: 389-398, 2007
43. Wang $X$ and Chan CB: $n-3$ polyunsaturated fatty acids and insulin secretion. J Endocrinol 224: R97-R106, 2015.

44. Rimoldi OJ, Finarelli GS and Brenner RR: Effects of diabetes and insulin on hepatic delta6 desaturase gene expression. Biochem Biophys Res Commun 283: 323-326, 2001.

45. Mansouri V, Javanmard SH, Mahdavi M and Tajedini MH: Association of Polymorphism in Fatty Acid Desaturase Gene with the Risk of Type 2 Diabetes in Iranian Population. Adv Biomed Res 7: 98, 2018.

46. Kim OY, Lim HH, Yang LI, Chae JS and Lee JH: Fatty acid desaturase (FADS) gene polymorphisms and insulin resistance in association with serum phospholipid polyunsaturated fatty acid composition in healthy Korean men: Cross-sectional study. Nutr Metab (Lond) 8: 24, 2011.

47. Yary T, Voutilainen S, Tuomainen TP, Ruusunen A, Nurmi T and Virtanen JK: Serum n-6 polyunsaturated fatty acids, $\Delta 5$ - and $\Delta 6$-desaturase activities, and risk of incident type 2 diabetes in men: The Kuopio Ischaemic Heart Disease Risk Factor Study. Am J Clin Nutr 103: 1337-1343, 2016.

48. Vessby B, Ahrén B, Warensjö E and Lindgärde F: Plasma lipid fatty acid composition, desaturase activities and insulin sensitivity in Amerindian women. Nutr Metab Cardiovasc Dis 22: 176-181, 2012.

49. Das UN: A defect in the activity of Delta6 and Delta5 desaturases may be a factor predisposing to the development of insulin resistance syndrome. Prostaglandins Leukot Essent Fatty Acids 72: 343-350, 2005.

50. Mathias RA, Pani V and Chilton FH: Genetic Variants in the FADS Gene: Implications for Dietary Recommendations for Fatty Acid Intake. Curr Nutr Rep 3: 139-148, 2014.

51. Wu Y, Ding Y, Tanaka Y and Zhang W: Risk factors contributing to type 2 diabetes and recent advances in the treatment and prevention. Int J Med Sci 11: 1185-1200, 2014.

52. Mani I and Kurpad AV: Fats and fatty acids in Indian diets: Time for serious introspection. Indian J Med Res 144: 507-514, 2016.

This work is licensed under a Creative Commons Attribution-NonCommercial-NoDerivatives 4.0 International (CC BY-NC-ND 4.0) License. 